22 日 (金) 第 4 会場

\title{
BCG maintenance therapy for non muscle invasive bladder cancer, from guidelines to practice : the french experience
}

\section{Department of Urology, Centre Hospitalier René Dubos, France} Patrick Coloby

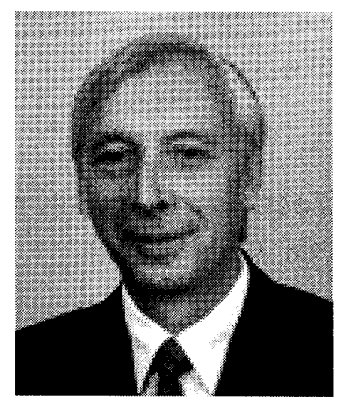

Context : Clinical practice guidelines (CPGs) provide an evidence based framework for clinical management that can help urologist decision-making, standardize best practice, and finally, enhance patient care and outcomes.

The French Urological Association (AFU) Clinical Practice Guidelines in Oncology are updated every 3 years and are based on the evaluation of scientific data and on expert opinion.

Concerning the high risk disease Non Muscle Invasive Bladder Cancer (NMIBC) group, french guidelines recommend a 6 week +3 week induction course of intravesical BCG and 1-3 year of maintenance BCG.

Objective : To develop strategies of disseminating and implementing CPGs in BCG therapy for Non Muscle Invasive Bladder Cancer (NMIBC) for their effective adoption in practice.

Methods : In order to evaluate the French urologists practice of BCG instillations in the treatment of non muscle invasive bladder cancer (NMIBC), a survey was performed, by AFU in 2008 using online self-administered questionnaires. Analysis of different factors who affect the natural or unaided adoption of guidelines was done in order to organize effective strategy by AFU.

Results : Although a majority of respondents said that first line plus maintenance BCG therapy was more efficient than first line alone, BCG maintenance therapy use and modality appeared to be heterogeneous.

Three factors appeared as an obstacle to use BCG maintenance. BCG therapy was not well accepted by patients $(46 \%)$, was not easy to organize $(28 \%)$ and was not well tolerated by patients $(56 \%)$.

Three actions were developped by AFU for urologists : patient education material to inform and help patient for a better treatment acceptation, reminders and pocket sized laminated cards to help urologist and nurse in care organization, practice prescription workshop to help urologist to prevent and to manage adverse effects.

Conclusion : appropriate implementation strategies of CPGs are important to reduce heterogeneous practice and to improve patient care.

\section{TRAINING AND DIPLOMA}

1974- graduation in medecine from the Paris VI University

1982 - specialist in Urology from the Paris VI University

\section{PROFESSIONAL EXPERIENCE}

Chief of the department of Urology since 1984.

Head of the department of Anesthesiology and Surgery since 2006.

President of the French Urological Association(AFU) from 2010.

Board member of the French College of Urology

Treasurer of the Société internationale d'Urologie(SIU).

\section{RESEARCH INTERESTS}

Clinical and basic research in urological oncology (specially in bladder cancer), urinary incontinence and BPH. 